\section{E.4 LAYOFFS AND THE MENTAL HEALTH AND SAFETY OF REMAINING WORKERS: A QUASI-EXPERIMENTAL STUDY OF THE U.S. ALUMINUM INDUSTRY}

'UC Berkeley School of Public Health Holly Elser*, ${ }^{2}$ Elijahu Ben-Michael, ${ }^{3}$ David Rehkopf, ${ }^{4}$ Sepideh Modrek, ${ }^{5}$ Ellen Eisen, ${ }^{6}$ Mark Cullen. ${ }^{1}$ UC Berkeley School of Public Health, Division of Epidemiology, Berkeley, USA; ${ }^{2}$ UC Berkeley, Department of Statistics, Berkeley, USA; ${ }^{3}$ School of Medicine, Department of Medicine, Division of Primary Care and Population Health Stanford University, Stanford, USA; ${ }^{4}$ Department of Economics, Health Equity Institute, San Francisco State University, San Francisco, USA; ${ }^{5}$ UC Berkeley School of Public Health, Division of Environmental Health Sciences, Berkeley, USA; ${ }^{6}$ Center for Population Health Sciences, Stanford University, Palo Alto, USA

\subsection{6/OEM-2019-EPI.82}

Background Relatively few studies have examined the effects of layoffs on remaining workers, although the effects of layoffs and downsizing events may extend beyond those employees who lose their jobs.

Methods We examined the effects of layoffs on mental healthcare utilization and injury risk among workers at 30 U.S. plants between 2003 and 2013. We defined layoffs as reductions in the hourly workforce of $20 \%$ or more at each plant. Using a difference-in-differences approach, we compared the change in outcomes during layoffs versus the same threemonth period one year previously, accounting for secular trends with control plants.

Results Our study population included 15502 workers and seven layoff events between 2003 and 2012. Layoffs were associated with decreases in reported injuries (-0.006, $95 \%$ CI $-0.013,0.001)$, and increased probability of outpatient visits $(0.010,95 \%$ CI $0.003,0.017)$ and prescriptions $(0.014,95 \%$ CI $-0.0006,0.027)$ for mental health. Among men, injury risk decreased more substantially (-0.010, $95 \%$ CI $-0.018,-0.001)$ and among women the increase in outpatient visits was more pronounced $(0.017,95 \% \mathrm{CI}$ $0.003,0.031)$. Most notably, the observed increase in prescription utilization appeared attributable primarily to increased frequency of opioid prescriptions (0.016 95\% CI 0.005-0.027).

Conclusion Our results indicate an association between layoffs and remaining workers' mental health and safety. However, decreased injury may reflect changes in reporting practices, and changes mental healthcare utilization may reflect changes in care-seeking. Future research on concordance of service utilization and underlying health and safety may yield valuable insight into the experiences employed workers in the wake of layoffs.

\section{E.5 MILITARY VETERANS PATHWAYS TO MENTAL HEALTH SUPPORT: THE NEED FOR NEW SOLUTIONS}

Laura Rafferty*, Sharon Stevelink, Neil Greenberg. King's College London, London, UK

\subsection{6/OEM-2019-EPI.83}

Military service can place personnel at a greater risk of developing mental health issues yet both serving personnel and military veterans are reluctant to seek help for mental health issues. Delay in seeking support for mental health distress can result in reduced quality of life as well as a worse treatment prognosis. This research aims to explore the barriers and facilitators to care for the UK veteran population, identifying the relationship between these factors and help seeking behaviour.
Sixty- two in-depth qualitative interviews were conducted with male UK military veterans who had left the Armed Forces in the last five years and screened positive for a degree of mental health distress on self-report questionnaires covering common mental disorders (anxiety or depression), post-traumatic stress disorder or alcohol misuse. Thematic analysis was utilised to identify core themes which were developed into an illustrative journey to mental health support, outlining the key stages through which a veteran may travel on their journey to engaging in effective mental health treatment.

Veterans' decision to seek care was mainly concentrated on the perceived need for treatment. Those not in mental health treatment failed to identify problems they were having as being indicative of a 'mental health disorder' as they had not yet reached a crisis point where they could no longer cope. Those veterans who were in mental health treatment typically had reached a point where the severity of their condition meant that their need for treatment was highlighted regardless of their intention, either due to a crisis event or to another's intervention.

Interventions are needed to target early identification and management of mental health distress and encourage veterans to seek support before reaching a crisis event. The implications for this research to other high stress, masculine occupations will be explored within the talk.

\section{O3E.6 ASSOCIATION BETWEEN WORKPLACE BULLYING AND COMMON MENTAL DISORDERS IN JUDICIARY BRAZILIAN CIVIL SERVANTS FROM SOUTHERN BRAZIL} 1,2,3Fernando Feijó* ${ }^{1}$ 'Anaclaudia Fassa, ${ }^{2}$ Neil Pearce. ${ }^{1}$ Federal University of Pelotas,
Pelotas, Brazil; ${ }^{2}$ London School of Hygiene and Tropical Medicine, London, UK; ${ }^{3}$ Federal
University of Recôncavo da Bahia, Santo Antônio de Jesus, Brazil

\subsection{6/OEM-2019-EPI.84}

Introduction Bullying has been described as a risk factor for mental disorders, however there is a lack of evidence on this subject in low- and middle-income countries. Therefore we aimed to evaluate the association between workplace bullying and common mental disorders in a sample of Judiciary Brazilian civil servants.

Methods Cross-sectional study with a sample of 1667 workers from the Brazilian Federal Judiciary in southern Brazil. The Negative Acts Questionnaire (NAQ-r) was used to measure bullying at work and the Self Report Questionnaire (SRQ-20) was used to evaluate Common Mental Disorders (CMD). Poisson regression was used to test associations of interest, controlling for confounders.

Results The overall prevalence of CMD was $33.5 \%$. The prevalence of workplace bullying was $17.7 \%$. In the crude analysis, exposure to bullying (at least one negative act in a weekly basis in the last six months) was associated with a 2.07-fold (CI 1.81-2.36, p<0.001) higher prevalence of CMD. After controlling for sociodemographic and occupational confounders, workers exposed to bullying presented a 1.51-fold (CI 1.31-1.73, $\mathrm{p}<0.001$ ) higher prevalence of CMD.

Discussion Judiciary Brazilian civil servants may be at a high risk of being exposed to bullying and having common mental disorders. Our findings corroborate the results of studies from high-income countries, where bullying is considered an 
important occupational determinant of mental health. Prevention policies to eliminate bullying in organizations are urgent, what may help to decrease the prevalence of mental disorders among workers.

\section{Shiftwork}

\section{A.1 NIGHT SHIFTWORK, DNA METHYLATION, AND TELOMERE LENGTH IN FEMALE NURSES}

${ }^{1}$ Michele Carugno*, 'Eleonora Crespi, 'Valentina Bollati, 'Letizia Tarantini, 'Laura Dioni, ${ }^{1}$ Luca Ferrari, ${ }^{1,2}$ Matteo Bonzini, ${ }^{2}$ Dario Consonni, ${ }^{1}$ Cristina Maggioni, ${ }^{1,2}$ Giovanni Costa,

${ }^{1,2}$ Angela Cecilia Pesatori. 'Dept. of Clinical Sciences and Community Health, Università degli Studi di Milano, Milan, Italy; 'Unit of Occupational Health, Fondazione IRCCS Ca' Granda Ospedale Maggiore Policlinico, Milan, Italy

\subsection{6/OEM-2019-EPI.85}

Introduction Studies on female nurses have reported a higher breast cancer risk among night shift (NS) workers, without a clear understanding of the underlying biological mechanisms.

Aim To assess the association between night shiftwork and molecular alterations potentially related to a higher carcinogenic risk, in details: DNA methylation of estrogen receptor (ER-Alpha, ER-Beta) and tumor suppressor (BRCA1, BRCA2, $\mathrm{p} 53$, p16) genes, global DNA methylation estimated on repeated elements (LINE-1, Alu), and telomere length (TL).

Methods 46 female nurses who had been working in NS for at least two years in a Hospital in Milan, Italy, were matched by age (30-45 years) and length of service (at least 5 years) with 51 female colleagues not working in NS. Each subject was administered a structured questionnaire and withdrawn a $12 \mathrm{ml}$ blood sample. We applied linear regression models adjusted for age, BMI, smoking habit, parity, and oral contraceptive use.

Results Currently working in NS (yes/no) was associated with hypomethylation of ER-Alpha ( $\beta$ : -1.635 , 95\% CI: -2.715 ; $-0.554)$. When examining both current and former NS workers, the number of years (NY) in NS was associated with hypermethylation of Alu ( $\beta$ : 0.078, 95\% CI: 0.016; 0.138). After graphical inspection of the association between NYNS and TL, we classified the study population according to NS duration ( $<15$ vs. $\geq 15$ years). Among workers with at least 15 years of NS, NYNS was associated with TL reduction ( $\beta$ : $-0.065,95 \% \mathrm{CI}:-0.122 ;-0.008)$ and hypomethylation of ER-Alpha ( $\beta$ : -2.009 , 95\% CI: $-3.164 ;-0.853)$. Association between NYNS and hypermethylation of p53, p16, BRCA1, BRCA2, and LINE-1 was much stronger, albeit not significant, in workers with at least 15 years of NS.

Conclusions Our findings show NS-associated epigenetic alterations that might be involved in processes such as cellular aging, genomic instability, and cancer development.

\section{O4A.2 JOINT EFFECTS OF NIGHT WORK AND SHIFT ROTATION ON TREATED DEPRESSION IN A LONGITUDINAL COHORT OF MANUFACTURING WORKERS}

${ }^{1}$ Jacqueline Ferguson*, ${ }^{1}$ Holly Elser, ${ }^{1}$ Sadie Costello, ${ }^{2}$ Mark Cullen, ${ }^{1}$ Ellen Eisen. ${ }^{1}$ UC Berkeley School of Public Health, Berkeley, USA; ${ }^{2}$ Stanford University, Stanford, USA

10.1136/OEM-2019-EPI.86
Past research consistently identifies shift work - a type of scheduled work that includes both night work and schedule rotations - as risk factor for depression. However, relatively few studies have examined whether people working more nights or rotations are more likely to seek treatment for depression.

A total of 5848 workers across 33 plants in the American Manufacturing Cohort (AMC) were followed 2003-2013. The first observed episode of treated depression was defined from insurance claims as two depression-related outpatient visits or two prescribed antidepressants within 365 days. Using detailed timeclock data, night work ( $\geq 3$ hours between 23:00 and 6:00) was defined as the percent of shifts that included a night shift and was categorized into non-night work $0 \%$; low >0\%-30\%; medium >30\%-70\%; and high 70\%-100\%. Shift rotations ( $\geq 6$ hours between subsequent shift start times), were similarly defined and classified as none $0 \%$, infrequent $<10 \%$, and frequent rotations $\geq 10 \%$. We examined the joint effects of night work and shift rotation on the time to first episode of treated depression using Cox proportional hazards regression.

Shift workers were more likely to be treated for depression compared with non-rotating, non-night workers. Workers with medium exposure to night work had the highest association with treated depression compared with permanent non-night workers, and the joint effects were similar between those with frequent rotations (HR: 1.64, 95\% CI: 1.00-2.67) and infrequent rotations (1.53, 0.992.37). Associations were slightly elevated among permanent night workers $(1.27,0.72-2.25)$ and workers with high exposure to night work regardless of rotations (1.36, $0.86-2.17)$.

Recent shift work exposure may be associated with higher rates of treated depression, and night work, rather than rotation, may be driving the association. However, there may be selection out of night work due to underlying depression and we were unable to differentiate new-onset from preexisting disease.

\section{A.3 DO SHIFT WORKERS HAVE A DIFFERENT EXPOSURE TO WORKPLACE CARCINOGENS THAN NON-SHIFT WORKERS?}

Sonia El-zaemey*, Renee Carey. Curtin University, Bentley, Australia

\subsection{6/OEM-2019-EPI.87}

Background There is limited information on whether the prevalence of exposure to workplace carcinogens varies among shift workers and non-shift workers.

Methods This analysis used data from the Australian Work Exposures Study-Cancer, a telephone survey which examined exposure to carcinogens in the workplace. Workers were classified as shift workers if they indicated that their usual roster ever included work between the hours of midnight and 5 am. Modified Poisson regression was used to estimate the adjusted prevalence ratios (aPRs) and 95\% confidence internals (CIs).

Results Among the 5425 workers, 6.88\% reported doing shiftwork. Overall, shift workers were more likely to be exposed to any carcinogen $(\mathrm{aPR}=1.16 ; 95 \% 1.06-1.26)$ and to multiple carcinogens $(\mathrm{aPR}=1.17 ; 95 \%$ 1.06-1.30) than non-shift workers. 